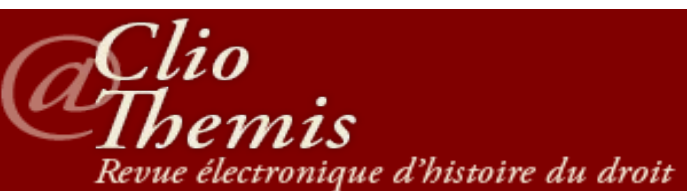

\title{
De quelle pensée juridique faisons-nous l'histoire ? Réflexions autour de quelques données bibliométriques
}

\begin{abstract}
Résumé: La lecture de la presse juridique du $\mathrm{XIX}^{e}$ siècle confronte l'historien de la pensée juridique contemporaine à un phénomène singulier: dans les colonnes des recueils d'arrêts figurent, en effet, de nombreux renvois à des œuvres et des auteurs oubliés par l'historiographie intellectuelle et littéraire du droit. Ne faudrait-il pas, dès-lors, redessiner la carte de la pensée juridique contemporaine et de sa littérature, sur le fondement de données bibliométriques significatives, pour raccrocher au mouvement des idées cette « foule des inconnus » qui a œuvré pour le droit ?
\end{abstract}

\begin{abstract}
The reading of the Nineteenth-Century's legal press confronts the bistorian of contemporary legal thought with a singular phenomenon: in the columns of this press, there are numerous references to works and authors forgotten by intellectual and literary legal historiography. Should we not then redesign the map of contemporary legal thought and of its literature, on the basis of significant bibliometric data, to hang on to the movement of ideas this « crowd of unknowns $\gg$ who worked for the law?
\end{abstract}

Mots-clés : Pensée juridique - histoire - bibliométrie - doctrine - pratique - jurisprudence

Keywords : Legal thought - bistory - bibliometrics - doctrine - practitioners - jurisprudence

\section{Genèse de cet article}

I. Au cours de nos travaux de thèse ${ }^{2}$, dans le souci d'exactitude et d'exhaustivité opiniâtre qui anime les recherches doctorales, nous avons effectué sur les recueils Sirey et Dalloz, entre i8oo et 1914, un recensement bibliographique aussi complet que possible, notant avec soin l'ensemble des ouvrages, articles, thèses et autres écrits cités par les arrêtistes dans leurs notes d'arrêts ou leurs notes de bas de pages.

2. Cette laborieuse collecte, exécutée sur plus de deux-cent-mille pages, était, à l'origine, destinée à fournir en annexe de ma thèse une bibliographie des ouvrages cités dans les recueils de jurisprudence sur le grand

I Nous tenons à remercier ici chaleureusement le Professeur Nader Hakim pour les discussions et échanges que nous avons eus ensemble et qui ont fait germer cette réflexion, ainsi que pour la réalisation du graphique bibliométrique général situé en fin d'article; ce travail chronophage et fastidieux, dont il s'est spontanément saisi, résume, en un schéma, tout l'intérêt de l'analyse bibliométrique dans l'étude de la pensée juridique et de son histoire. Nous voudrions également remercier, tout aussi chaleureusement, les Professeures Anne-Sophie Chambost et Géraldine Cazals pour leurs relectures, leurs conseils et leurs retours encourageants.

2 P.-N. Barenot, Entre théorie et pratique : les recueils de jurisprudence, miroirs de la pensée juridique française (I789-I9I4), sous la direction de M. le Professeur Nader Hakim, thèse soutenue le 7 novembre 20I4, en projet de publication. 
$\mathrm{XIX}^{\mathrm{e}}$ siècle. Pour diverses raisons, cette annexe n’a pas été intégrée à la production finale, mais nous avons néanmoins conservé ces données pour les exploiter ultérieurement.

3. Un élément à l'époque avait toutefois retenu notre attention : de très nombreux auteurs, qui nous étaient parfaitement inconnus - ou peu connus -, étaient régulièrement cités comme autorités par les rédacteurs de ces recueils, parfois plusieurs décennies durant; leurs ouvrages et travaux apparaissaient comme incontournables, et constituaient des références, des sources de réflexions et d'inspiration pour les arrêtistes. À l'inverse, les «grands noms » de la doctrine du XIX siècle, ceux sur lesquels, en somme, s'affairent les historiographes de la pensée juridique contemporaine depuis les travaux fondateurs de Julien Bonnecase ou d'Eugène Gaudemet's, nous semblaient bien plus modestement référencés au sein de ces grands recueils.

4. Ce constat était particulièrement évident jusqu'aux années I88o, tournant à partir duquel les recueils de jurisprudence, initialement fondés et dirigés par des praticiens, ont été massivement investis par les universitaires qui en ont progressivement pris le contrôlet. Dès lors, les auteurs et les travaux cités par les professeurs-arrêtistes dans les recueils de la Belle Époque prirent une coloration nettement plus académique, pour ne pas dire « autocentrée », et nous apparurent, en conséquence, plus familierss.

5. Que penser toutefois de cette masse d'œuvres et d'auteurs manifestement plébiscités par les arrêtistespraticiens sur les deux-tiers du XIX siècle, et dont l'histoire n'a pourtant gardé trace ? Si l'on s'en tient à l'acception première du terme, il s'agit d'œuvres de «doctrine », définie par Marcel Planiol comme les «opinions et les idées émises par les jurisconsultes dans leurs ouvrages $»^{6}$, leurs auteurs étant, pour l'essentiel, des praticiens du droit. Cependant, il semblait quelque peu délicat de parler - sans autres précisions -, de doctrine à propos de ces ouvrages, puisque la doctrine, pour être désignée comme telle, implique une certaine reconnaissance épistémique de la part... de la doctrine elle-même ${ }^{7}$ !

6. En effet, depuis la Belle Époque au moins, la doctrine est universitaire et répond à un triptyque implacable, même s'il n'est pas toujours clairement affiché ou assumé : est une ouvre de doctrine une œuvre de droit dogmatique ${ }^{8}$, rédigée par un universitaire (ou assimilé), dont les pairs ont reconnu l'autorités. Ainsi, pour incorporer la doctrine, le statut et l'intégration académique de l'auteur

3 J. Bonnecase, La Thémis (I8Ig-I83I): son fondateur, Athanase Jourdan, Paris, Société du recueil Sirey, I9I4 ; Id., La pensée juridique de 1804 à l'heure présente: ses variations et ses traits essentiels, Bordeaux, Delmas, 1933; E. Gaudemet, L'interprétation du code civil en France depuis I804, conférences données à l'Université de Bâle, le 3o novembre, 7, I4 et 21 décembre 1923 [Préface de A. Simonius.], Paris, Librairie du recueil Sirey, 1935; rééd., L’interprétation du Code civil en France depuis 1804, Présentation de C. Jamin et P. Jestaz, bibliographie critique par F. Rolin, Paris, Collection du deuxième centenaire du Code civil, La Mémoire du Droit, Dalloz, 2002.

4 P.-N. Barenot, Entre théorie et pratique, op. cit., p. 276 et suiv.

5 En effet, la doctrine de « l'École scientifique » et des auteurs du « moment 1900 » est certainement la plus étudiée par les historiens de la pensée juridique contemporaine. Pour un aperçu suffisamment illustratif, v. N. Hakim et F. Cherfouh, «L'histoire de la pensée juridique contemporaine, hétérogénéité et expansion », dans L'Histoire du droit en France. Nouvelles tendances, nouveaux territoires, B. d'Alteroche et J. Krynen (dir.), Paris, Garnier, 20I4, p. II7-I43, et plus spécialement p. 129 et suiv.

6 M. Planiol, Traité élémentaire de droit civil, Paris, F. Pichon, I900, t. I, n 42, p. I4.

7 Voir notamment sur ce point C. Jamin et P. Jestaz, La doctrine, Dalloz, coll. méthodes du droit, 2004 ; É. Millard, « Ce que "doctrine" veut dire », dans La doctrine en droit administratif, Paris, Travaux de l'Association française pour la recherche en droit administratif, Litec, 20I0, p. 3-I2.

8 Voir notamment C. Jamin, « La construction de la pensée juridique française : interrogations sur un modèle original à l'aune de son anti-modèle », dans L'architecture du droit. Mélanges en l'honneur de Michel Troper, D. de Béchillon, P. Brunet, V. Champeil Despats et É. Millard (dir.), Paris, Economica, 2006, p. 50I-517 ; N. Hakim, « Droit privé et courant critique : le poids de la dogmatique juridique $\gg$, dans Le droit en révolution(s) - Regards sur la critique du droit des années 1970 à nos jours, X. Dupré de Boulois et M. Kaluszinsky (dir.), Paris, Droit et société, Maison des Sciences de l'Homme Réseau Européen Droit et Société, Librairie générale de droit et de jurisprudence, 20II, p. 7I-83.

9 Ce schéma s'applique naturellement à la doctrine civiliste, voire privatiste dans son ensemble, ainsi qu'à une grande partie de la doctrine publiciste ; voir notamment N. Hakim, Lautorité de la doctrine civiliste, Paris, Librairie générale de droit et de jurisprudence, 2004. En revanche, le cas de la doctrine administrativiste est à part, puisque les universitaires n'ont jamais réussi à s'en arroger le monopole ; cette doctrine « dyarchique » est donc partagée avec les membres du Conseil d'État, qui, peut-être, la marquent plus fortement encore de leur autorité déontique. V. notamment J. Rivero, « Apologie pour les 
(titularisation, grade, agrégation, responsabilités scientifiques, fonctions de direction, etc.) comptent autant que la force de ses travaux ${ }^{10}$. Les dits travaux doivent, en outre, correspondre à des genres doctrinaux identifiables" et ne pas trop sortir des canons de la dogmatique, sous peine de ne plus être considérés comme $\ll$ de la doctrine ${ }^{12}$.

7. Pour en revenir aux écrits référencés dans les recueils Sirey et Dalloz du XIXe siècle, ces derniers ne sont pas, pour l'essentiel, des travaux de professeurs, mais des travaux de praticiens ; surtout, l'historiographie de la pensée juridique - écrite par l'École -, ne les a jamais élevés au rang des œuvres de la doctrine dite « classique », celle qui va des premiers commentaires du Code civil jusquaux travaux du « renouveau » de l'École scientifique, au tournant du XIX ${ }^{e}$ siècle $^{13}$. Pour toutes ces raisons, il nous semblait donc délicat de parler de doctrine à leur sujet.

8. Le terme de « doctrine praticienne » nous paraissait alors plus approprié, mais il n'était pas non plus pleinement satisfaisant. En effet, la « pratique » est polysémique en droit ${ }^{14}$, et certains des auteurs cités par les arrêtistes n'étaient pas des praticiens. En outre, si la distinction doctrine universitaire/doctrine praticienne pouvait se révéler commode, elle ne répondait à aucune question de fond : en quoi cette « doctrine de praticiens » était-elle différente de celle des universitaires ? Était-elle véritablement moins théorique ? Moins dogmatique ? Moins inspirée ou inspirante ? Ne s'adressait-elle vraiment qu'aux seuls praticiens du droit ? Cette dichotomie un peu artificielle entre classes d'auteurs et d'œuvres ne permettait donc pas d’appréhender la réalité de cette littérature dite « praticienne », ni de comprendre pourquoi cette dernière n'avait, jusqu'à lors, suscité que si peu d'intérêt auprès des historiographes.

9. Plutôt donc que de parler de doctrine praticienne à propos de ces auteurs et de leurs œuvres, il nous semble préférable de parler aujourd'hui d'une pensée juridique contemporaine encore inexplorée. En effet,

"faiseurs de systèmes" », Dalloz, I951, chronique, p. 99-IO2; M. Deguergue, Jurisprudence et doctrine dans l'élaboration du
droit de la responsabilité administrative, Paris, Librairie générale de droit et de jurisprudence, Paris, I994; J. Krynen,
«Conclusions », dans Les facultés de droit inspiratrices du droit, Toulouse, Travaux de l'Institut fédératif de recherche
«Mutation des normes juridiques », $\mathrm{n}^{\circ} 3$, 2005, p. 227-234; J.-L. Mestre, «L'histoire du droit administratif », dans Traité
de droit administratif, P. Gonod, F. Melleray et P. Yolka (dir.), t. I, Paris, Dalloz, 20II, p. 3-58; v. aussi A. Ciaudo, « Retour
sur quelques paradoxes: le juge administratif et la doctrine du droit administratif » [en ligne:
http://www.blogdroitadministratif.net/, 2007].

Io C'est pourquoi, notamment, la thèse d'un jeune docteur, les articles d'un doctorant ou d'un « post-doc », aussi académiques ou profonds soient-ils, seront sans difficultés qualifiés de travaux «scientifiques », mais jamais de « doctrine ». Voir contra É. Millard, « Ce que "doctrine" veut dire », art. cit.

II N. Hakim, « Les genres doctrinaux », dans La doctrine en droit administratif, op. cit., p. I47-I68.

I2 C. Jamin et P. Jestaz, La doctrine, op. cit.

I3 Cela ne saurait, bien évidemment, signifier que les historiens de la pensée juridique contemporaine ne se soient jamais écartés de cette doctrine pour explorer la pensée d'auteurs plus atypiques ou de praticiens! Voir notamment la thèse récemment publiée de F. Cherfouh, Le juriste entre science et politique La revue générale du droit, de la législation et de la jurisprudence en France et à l'étranger (1877-1938), Paris, Librairie générale de droit et de jurisprudence, 2017. Néanmoins, cet « axe doctrinal » demeure central dans les études historiographiques.

I4 Voir notamment les travaux de J. Hilaire : « Pratique notariale et influence universitaire à Montpellier au Moyen-Âge », dans Mélanges André Dupont, Montpellier, Fédération historique du Languedoc méditerranéen et du Roussillon, 1974, p. I67-18I ; J. Hilaire, J. Turlan et M. Villey, « Les mots et la vie - la "pratique" depuis la fin du Moyen-Âge », dans Droit privé et institutions régionales - Études historiques offertes à Jean Yver, Paris, Presses universitaires de France, 1976, p. 369387 ; J. Hilaire, «Actes de la pratique et expression du droit », Droits, Revue française de théorie, de philosophie et de cultures juridiques, 7, 1988, p. I35-I4O ; «pratique », dans Dictionnaire de la culture juridique, D. Alland et S. Rials (dir.), Paris, Presses universitaires de France, p. II80-II85; C. Chêne, « Pigeau et Bellart, la formation des praticiens du droit de la fin de l'Ancien Régime à la Restauration », dans Les praticiens du droit du Moyen Age à l'époque contemporaine : approches prosopographiques, Belgique, Canada, France, Italie, Prusse, V. Bernaudeau, J.-P. Nandrin et B. Rochet (dir.), actes du colloque de Namur, I4, I5 et 16 décembre 2006, Rennes, Presses universitaires de Rennes, 2008, p. $269-278$. Comme l'a démontré Jean Hilaire, avocats et magistrats sous l’Ancien Régime ne faisaient pas de la « pratique », mais du «droit ». La pratique désignait alors très précisément la procédure, dont était en charge le procureur. Le juge, l'avocat, l'avoué ou même le notaire de l'Ancien Droit n'étaient donc pas à proprement parler des praticiens. La véritable scission entre les «praticiens du droit » et les «théoriciens du droit » ne se fera qu'au XIX siècle, et pas de façon tranchée, avec l'éclatement de la figure du « jurisconsulte ». 
la pensée juridique est plurielle ${ }^{\text {Is }}$; nous n'écrivons pas l'histoire de la pensée juridique, mais l'histoire « des pensées juridiques $»^{16}$, pour ne pas dire « des histoires $\gg$ de la pensée juridique ${ }^{17}$.

Io. Jusqu'à présent, force est de constater que les travaux historiographiques se sont, pour l'essentiel, concentrés sur la doctrine, la doctrine ayant elle-même initié ces études à la fin du XIX siècle. À ce titre, et c'est aujourd'hui bien connu, les historiographes de l' «École scientifique » ont écrit l'histoire de la doctrine qui l'a précédée dans une optique d’autopromotion, n’hésitant pas à expliquer en quoi leurs travaux étaient remarquablement plus rigoureux et «scientifiques » que ceux de leurs prédécesseurs ${ }^{18}$. Dans leurs études, ces derniers ont, en outre, écarté ou minimisé un certain nombre de travaux, généralement issus d'hommes du Palais - des ouvres donc « pratiques » et non « doctrinales »-, qui ont pourtant marqué la littérature juridique de leur siècle. Et si les chercheurs ont désormais largement réinvesti et réhabilité les travaux de l'École dite de l' « exégèse $»^{19}$, s'ils continuent à explorer l'œuvre monumentale de la doctrine de la Belle Époque et de l'Entre-Deux Guerres, ils se sont en revanche encore peu aventurés hors des «sentiers battus » de la doctrine, de la pensée juridique universitaire. Or, cette histoire de la pensée juridique universitaire ne saurait être tenue que pour une partie seulement de la pensée juridique contemporaine ; biens d'autres sentiers et chemins demeurent à explorer pour espérer dresser un jour la cartographie complète de cette pensée juridique plurielle, et l'étude de la pensée juridique « non doctrinale » constitue une de ces pistes à ouvrir.

II. Toutefois, comment recenser et explorer efficacement cette pensée juridique mal connue ou inconnue ? Un des moyens envisageables pour en obtenir un aperçu suffisamment représentatif pourrait être d'utiliser les outils de la bibliométrie ${ }^{20}$.

\section{Réflexions sur la méthode employée}

Is Voir sur ce point O. Jouanjan, Une bistoire de la pensée juridique en Allemagne (I800-IgI8), Paris, Presses universitaires de France, 2005 , p. 2. Plurielles, les pensées juridiques s'inscrivent également - et plus largement - au sein d'ensembles distincts de discours, de représentations et de pratiques, caractérisant autant de cultures juridiques. L'approche culturelle des savoirs juridiques demeure là encore un grand chantier, que les travaux récents de Frédéric Audren et de Jean-Louis Halpérin ont brillamment ouvert (F. Audren et J.-L. Halpérin, La culture juridique française, entre mythes et réalités XIX ${ }^{e}$ $X X^{e}$ siècle, Paris, coll. CNRS Droit, 2013).

I6 N. Hakim et F. Cherfouh, « L'histoire de la pensée juridique contemporaine », art. cit., p. I22.

I7 C'est pourquoi nous parlons ici de « la » doctrine, au singulier, nonobstant la multitude de sujets (forcément juridiques) qu'elle est susceptible d'aborder, de positionnements qu'elle est susceptible d'adopter, et de ses transformations à travers

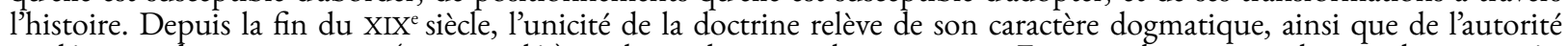
académique des universitaires (ou assimilés) qui la produisent et la composent. En revanche, nous parlons « des » pensées juridiques, au pluriel; si le concept de pensée est globalisant, nous voulons insister par ce pluriel sur le caractère hétéroclite des auteurs susceptibles de développer une pensée juridique (juristes comme non juristes, praticiens du droit, historiens, philosophes, politiques, écrivains, etc.), ainsi que sur le vaste panel des modes d'expression de cette pensée, qui déborde très largement des genres habituellement admis par la doctrine (essai, roman, pamphlet, littérature de vulgarisation, etc.).

I8 Voir notamment les œuvres d'Eugène Gaudemet et de Julien Bonnecase précitées; aussi C. Jamin, « L'oubli et la science, regard partiel sur l'évolution de la doctrine privatiste à la charnière des $\mathrm{XIX}^{\mathrm{e}}$ et $\mathrm{XX}^{\mathrm{e}}$ siècles $\gg$, Revue trimestrielle de droit civil, 1994 , p. $815-827$.

19 Citons, entre-autres, l'article fondateur de P. Rémy, «Éloge de l'exégèse », Revue de la recherche juridique, 1982, p. 254262, repris dans Droits, Revue française de théorie, de philosophie et de cultures juridiques, 1985, p. II5-I23; id., « Le rôle de l'exégèse dans l'enseignement du droit au XIX siècle », Annales des facultés de droit, 1985-2, p. 9I-IO5; pour une étude plus générale, J.-L. Halpérin, Histoire du droit privé français depuis I804, 2éd., Paris, Presses universitaires de France, 2012.

20 Entendue ici dans son sens le plus large, comme « l'application des mathématiques et des méthodes statistiques aux livres, articles et autres moyens de communication », v. A. Pritchard, «Statistical Bibliography or Bibliometrics », Journal of Documentation, 25, 4, 1969, p. 348-349. Sur la bibliométrie, ses méthodes et son application au champ de l'histoire de la pensée juridique, nous nous permettons de renvoyer à l'article de N. Hakim et A. Monti publié dans ce numéro de Clio@Thémis, «Circulation des idées juridiques et analyse bibliométrique : l'exemple franco-italien du droit des assurances $\gg, 14,2018$, http://www.cliothemis.com/Circulation-des-idees-juridiques. 
I2. Il pourrait y avoir quelque paradoxe à inviter à la bibliométrie tout en relevant, dans le même temps, le phénomène d'autocitation exclusiviste de la doctrine, qui conduit à dissimuler des pans entiers de la production littéraire du droit et de la pensée juridique. En effet, lorsque l'Université s'empare du monopole de la science - autrement dit de la «doctrine »-, au tournant des années I88o, les citations d'articles et d'ouvrages, au sein d'une littérature juridique et d'un univers éditorial qu'elle domine désormais, ne renvoient presque exclusivement plus qu'à la doctrine elle-même, la science fonctionnant en réseau, citations et références reliant entre eux les chercheurs, les « écoles » et les institutions ${ }^{21}$.

I3. Toutefois, avant la Belle Époque, le monde de l'édition juridique n'est pas encore submergé par les plumes de l'École; les maisons Sirey et Dalloz, pour ne citer qu'elles parmi les principales et les plus influentes, se sont d'ailleurs construites autour de recueils de jurisprudence, périodiques rédigés par - et pour - les praticiens du droit. Si nous manquons d'études générales portant sur les fonds de bibliothèques d'avocats ou de juridictions au XIX ${ }^{e}$ siècle, et si l'analyse des listes de souscription aux grands périodiques juridiques nous éclairerait grandement sur la réalité de leur lectorat ${ }^{22}$, nous pouvons raisonnablement penser que les recueils d'arrêts et les journaux assimilés étaient majoritairement consultés par les hommes du Palais. La lecture des recueils Sirey et Dalloz ne laisse d'ailleurs que peu de place au doute : avant I88o, ce sont presque uniquement des avocats et des magistrats qui y écrivent ; en outre, la place qu'y tiennent les questions de procédure ou les sujets professionnels (notariat, avoués, questions ordinales) est révélatrice d'une culture juridique que, faute de mieux, nous qualifierons ici de « praticienne ».

I4. L’analyse bibliométrique sur ces recueils, notamment sur la période antérieure à i88o, nous donne alors un aperçu des auteurs et des ouvrages connus, lus et recommandés par les praticiens du droit. En d'autres termes, cette analyse constitue une voie d'accès relativement objective ${ }^{23}$ à la pensée juridique dominante chez les praticiens du XIX siècle, pensée juridique qui n'est pas celle de la doctrine, ni celle dépeinte $a$ posteriori par l'Université à partir de la Belle Époque.

I5. Les données qui suivent sont issues du recueil Dalloz, sur la période allant de I825 à $1914^{24}$. Le nombre total d'auteurs cités sur cet intervalle de 89 ans est bien entendu beaucoup trop grand pour être exploitable dans le cadre d'un article. Il a donc fallu trouver des critères pour écarter les auteurs jugés « secondaires », c'est-à-dire ceux qui n’apparaissent pas assez souvent dans les colonnes pour être considérés comme de véritables « autorités ».

16. C'est là, sans aucun doute, le point faible de notre essai d'analyse bibliométrique, dans le sens où des critères plus travaillés auraient certainement donné des résultats plus fins, et possiblement différents. Alors que nous venions de dépouiller intégralement le recueil Dalloz, il nous avait semblé, à l'époque, préférable d'exclure tous les auteurs qui n'avaient pas été cités, durant au moins cinq années, sur l'une au moins des huit périodes suivantes : I825-1835; I836-1847; I848-1858; I859-1869; I870-1880 ; I88I-I89I ; I892-1902 et 1903-1914 ${ }^{25}$.

2I V. R. K. Merton, The sociology of science, theorical and empirical investigations, Chicago, The University of Chicago Press, 1973; B. Latour, La science en action, Paris, Gallimard, Folio Essais, 2005; A. Saint-Martin, La Sociologie de Robert K. Merton, Paris, La Découverte, coll. « Repères », 2013.

22 À supposer que de telles listes aient existé, et qu'elles aient été conservées par les éditeurs (après fusions ou rachats) ou au sein dautres archives privées. La réalité du réseau de diffusion de ces périodiques est également une inconnue aujourd'hui. Ici encore, les recherches sur l'histoire éditoriale et commerciale de la littérature juridique constituent un immense chantier. V. sur ce point les travaux de Jean-Yves Mollier, de Valérie Tesnière, d’Anne-Sophie Chambost et de Géraldine Cazals.

23 Bien entendu, ces résultats ne doivent pas dissimuler les enjeux commerciaux qui demeurent derrière la citation d'ouvrages. Les périodiques mettent - aussi - en avant dans leurs colonnes les œuvres de leurs collaborateurs, ou celles qui sont éditées par leurs soins.

24 En I822, Désiré Dalloz prend la direction, avec le greffier Tournemine, du Journal des Audiences, périodique de jurisprudence fondé en l'An XII par le greffier Géraud-Thimothée Denevers. Mais c'est en i825 que Dalloz renomme ce journal Jurisprudence générale du royaume, en matière civile, commerciale et criminelle; cette date marque ainsi le véritable acte de naissance du célèbre « Dalloz Périodique ».

25 Ce découpage par «tranches » de dix ans a l'avantage de concorder avec plusieurs grandes périodes de la pensée juridique contemporaine et de l'histoire éditoriale de la revue. 
I7. Si ce critère peut paraittre arbitraire - et, dans une certaine mesure, il l'est -, il nous avait néanmoins permis de ne garder en lice que les auteurs dont la pérennité, et donc l'autorité au recueil, étaient forte. En outre, la liste dressée reflétait assez bien nos impressions de lecture, les noms relevés étant ceux qui défilaient invariablement dans le recueil Dalloz, et qui avaient fini par s'inscrire dans notre mémoire, pages après pages, volumes après volumes.

I8. Il nous faut apporter ici une dernière précision importante : nous avons exclu de cette liste tous les renvois aux auteurs d'Ancien Régime ainsi que les renvois au droit de Justinien, encore assez nombreux dans le premier tiers du XIX ${ }^{e}$ siècle. Cette exclusion avait deux principales raisons : d'une part, nous ne voulions recenser que les auteurs contemporains des recueils; d'autre part, après quelques années de questions transitoires, les autorités d'Ancien Droit ne furent que rarement utilisées pour résoudre des difficultés positives, mais servirent la plupart du temps d’appuis secondaires - et quelque peu érudits -, à des argumentaires structurellement fondés sur la loi, la jurisprudence et les opinions des auteurs contemporains ${ }^{26}$.

19. La liste totale des autorités retenues suivant ces critères relativement stricts s'élève, tout de même, à 56I noms. Dans le cadre de cet article, il s'agit simplement de mettre en avant quelques données bibliométriques parmi les plus significatives.

\section{Les données, et ce qu'elles suggèrent}

20. Les relevés bibliométriques effectués ici sont très sommaires, et ne donnent qu'un aperçu embryonnaire du travail qui pourrait être effectué à plus grande échelle, avec un niveau de précision supérieur et des outils plus adaptés ${ }^{27}$. Les principales forces de l'analyse bibliométrique, c'est-à-dire la mise en réseau des auteurs, l’analyse à plusieurs niveaux des objets (sources, revues, pays, institutions, etc.) ou encore l'établissement de facteur d'impacts (ou ses modèles alternatifs) n'ont pas été déployées ici. Pour cela, il faudrait au préalable effectuer un recensement d'ampleur sur les grandes revues juridiques ainsi que sur l'essentiel des travaux publiés sur le siècle ${ }^{28}$, afin d'en tirer des résultats généraux qui livreraient une photographie tout à fait neuve de la pensée juridique contemporaine.

2I. Beaucoup plus modestes, nos relevés bibliométriques nous ont toutefois permis de mettre en lumière quelques réalités qui échappent aux premières impressions ou aux présupposés.

22. Ainsi, sans être représentatifs à eux seuls de la pensée juridique dominante du recueil Dalloz au $\mathrm{XIX}^{\mathrm{e}}$ siècle, 6 auteurs sur 56I se retrouvent cités, au moins une fois par volume, entre i825 et I9I4; en d'autres termes, on retrouve leurs noms et leurs œuvres sans discontinuer au recueil, année après année, sur une période de 89 ans!

23. Il s’agit de Dalloz, Duranton, Locré, Merlin de Douai, de Pardessus et de Toullier.

24. Retrouver le nom de Dalloz au sein de cette liste n'est pas surprenant; en effet, le recueil périodique est un excellent media d'autopromotion. Les ouvres des frères Dalloz - le Répertoire dirigé par Désiré, et le

26 V. sur ce point particulier l'étude de N. Hakim, « Continuité ou rupture dans l'histoire de la pensée juridique ? Exégèse, transtextualité et positivisme du Cours de Code Napoléon de Charles Demolombe », dans Mélanges en l'honneur de JeanLouis Thireau, A. Dobigny-Reverso, X. Prévost et N. Warembourg (dir.), Paris, Mare\&Martin (Collection de l'Institut des sciences juridique et philosophique de la Sorbonne), à paraître.

27 Notamment en ce qui concerne la création, la gestion de bases de données, puis leur exploitation graphique (via le logiciel libre Gephi, $c f$. le graphique bibliométrique général en fin d’article, ou encore via la plateforme Nodegoat).

28 Il existe déjà pour cela des ressources, comme la Bibliographie générale des sciences politiques, juridiques, économiques et sociales de I80o à 1925-26 d’A. Grandin (Paris, Sirey, 1926). 
Dictionnaire rédigé par Armand ${ }^{29}$ - mais aussi les nombreux Codes annotés, publiés par la maison et régulièrement mis à jour, sont naturellement mis en avant dans les colonnes du périodique.

25. Le cas de Duranton et de Toullier peut surprendre davantage. En effet, ces deux auteurs figurent parmi les premiers commentateurs du Code, et, si leurs œuvres ont, sans conteste, connu un grand succès, les travaux ultérieurs d'Aubry et Rau, de Demolombe, de Troplong ou encore de Laurent auraient pu les surclasser par la suite, en matière de référencement. Duranton et Toullier demeurent néanmoins inlassablement cités chaque année au recueil, et ce jusqu'en I9I4!

26. Deux nuances doivent toutefois être apportées ici : les autres grands commentateurs classiques sont également très présents dans les citations, et certains d'entre eux, comme Proudhon, arrivent au même degré de référencement si on prend en compte les travaux de leurs continuateurs; Curasson, Dumay et Valette continueront, chacun de leur côté, l'œuvre de Proudhon, tandis que Colmet de Santerre perpétuera l'œuvre de Demante, prolongeant ainsi le référencement et l'autorité des travaux de leurs aînés.

27. Le cas de Locré illustre pour sa part la permanence d'une grande règle de la méthode exégétique, qui ne disparaîtra pas avec l'essor de « l'Ecole scientifique » et de la « libre recherche scientifique » préconisée par François Gény : « quand la loi est obscure, il faut en approfondir les dispositions pour en pénétrer l'esprit et rechercher l'intention du législateur, en se référant notamment aux travaux préparatoires ». Les procès-verbaux du Conseil d'État rassemblés par Locré au tout début du XIX siècle lors des discussions sur les codes civil, commercial et criminel, constituent ainsi, presque un siècle plus tard, une base intarissable d'arguments et d'éclaircissements pour interpréter la loi (mais aussi, d'arguments ou d'illustrations historiques).

28. En ce qui concerne Merlin de Douai, son Répertoire et ses Questions de Droit, maintes fois réédités jusqu'aux années I830, constituent des œuvres majeures de la lexicographie juridique. On imagine sans mal que ces ouvrages étaient particulièrement bien implantés dans les bibliothèques comme dans la culture juridique des praticiens du droit; en continuant le célèbre Répertoire de son maître Guyot, Merlin a accompagné et éclairé les praticiens à l'aube du XIX siècle dans les questions transitoires, du droit ancien au droit intermédiaire et au droit codifié. Considéré comme un véritable « oracle » par l'arrêtiste concurrent Jean-Baptiste Sirey, Merlin de Douai est l'un des auteurs les plus cités dans les recueils de jurisprudence, et ses travaux lexicographiques ne seront que lentement et tardivement supplantés par les grands Répertoires généraux de Dalloz, de Favard de Langlade ou encore de Fuzier-Hermann.

29. On peut toutefois s'étonner de trouver encore le nom de Merlin de Douai à la fin du XIXe et au début du XXe siècle. À cette époque, l'œuvre de l'auteur n'est naturellement plus invoquée pour résoudre des difficultés de droit transitoire ou pour inspirer une jurisprudence encore balbutiante ; elle constitue désormais un «monument», une synthèse facile à manœuvrer de droit ancien et contemporain, de théorie et de pratique, permettant - sans trop d'efforts - d'élaborer des généalogies juridiques ou d'introduire une note d'arrêt par des éléments historiques.

30. Pardessus, enfin. S’il n'est pas un auteur ignoré des historiographes, le commercialiste n’a pas suscité autant d'intérêt de la part des chercheurs qu'un Thaller ou un qu'un Lyon-Caen. Pourtant, son Cours de droit commercial - et, dans une moindre mesure, son Traité des servitudes et sa Collection de lois maritimes - traversent le siècle, et sont encore très référencés à la Belle Époque, alors que l'École se dote de grands commercialistes. Cette donnée pourrait inviter à davantage se pencher sur l'œuvre de Pardessus, et sur son influence dans la pratique et dans la pensée commercialiste contemporaine.

3I. Plus représentatifs de la pensée juridique du recueil Dalloz, 58 auteurs ${ }^{30}$ ont été cités au moins une fois sur chaque décennie, entre i825 et I9I4. Leur référencement peut-être très variable d’une décennie à l'autre,

29 D. Dalloz, Répertoire méthodique et alphabétique de législation, de doctrine et de jurisprudence, en matière de droit civil, commercial, criminel, administratif, de droit des gens et de droit public, etc., Ire éd., Paris, Bureau de la jurisprudence générale, 1824-I830; A. Dalloz, Dictionnaire général et raisonné de Législation, de Doctrine et de Jurisprudence en matière civile, commerciale, criminelle, administrative et de droit public, etc., Paris, Bureau de la jurisprudence générale, I835-I84I. 
et parfois même d'une année sur l'autre; néanmoins, ces jurisconsultes et leurs travaux demeurent des autorités particulièrement pérennes au sein du recueil; ils constituent donc le fond intellectuel et bibliométrique dominant du recueil Dalloz sur le grand XIX ${ }^{e}$ siècle.

32. On retrouve ces 58 auteurs dans le graphique de synthèse figurant ci-dessous (schéma I). Ce dernier a été automatiquement généré à l'aide des données collectées et entrées dans un fichier Excel ${ }^{\circledR}$; à chacune des huit périodes, le logiciel a attribué automatiquement un code couleur. Au-dessus des auteurs, les indices de I à 3 correspondent à la fréquence de citation de l'auteur sur chaque intervalle : I signifie que l'auteur a été cité dans le recueil sur une à quatre années par période (« faible »); 2 signifie qu'il a été cité de cinq à neuf années (《moyenne »); 3 signifie qu'il a été cité sur dix années ou plus, c'est-à-dire sur l’intégralité de la période concernée (《 forte »).

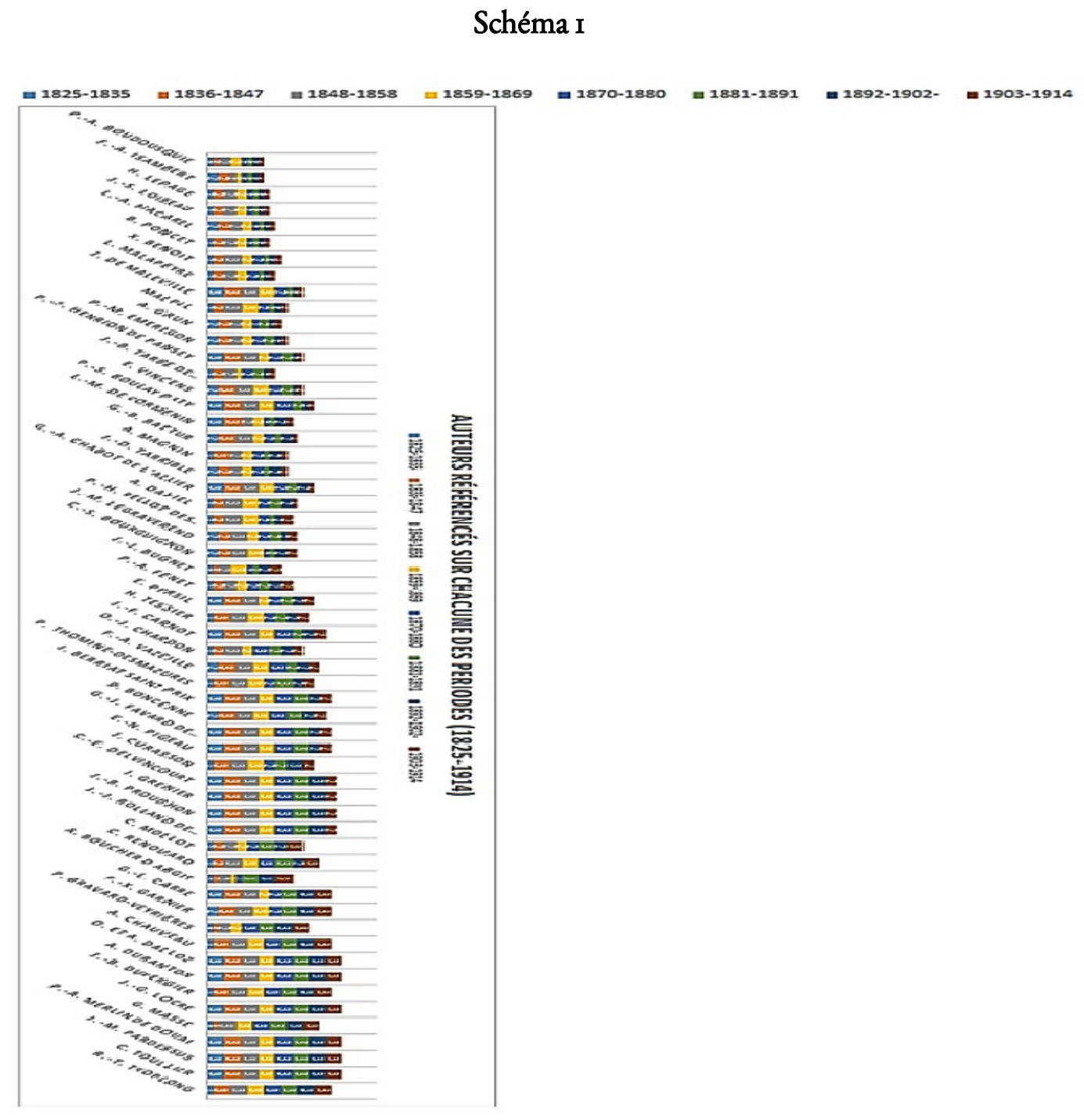

30 Il s'agit de: P.-A. Boudousquie; F.-A. Isambert ; H. Lepage ; J.-S. Loiseau ; L.-A. Macarel ; B. Poncet ; X. Benoit ; L. Malapeyre; J. de Maleville; Malpel; A. Grun ; B.-M. Émerigon; P.-P. Henrion De Pansey; J.-B. Tarbe de Vauxclairs; E. Vincens; P.-S. Boulay Paty; L.-M. de Cormenin; G.-B. Battur; A. Magnin ; J.-D. Tarrible ; G.-A. Chabot De Lallier ; A. Daviel; P.-H. Bellot Des Minieres ; J.-M. Legraverend; C.-S. Bourguignon ; J.-J. Bugnet ; P.-A. Fenet ; E. Persil ; H. Tessier ; J.-F. Carnot ; O.-J. Chardon ; F.-A. Vazeille ; P. Thomine-Desmazures ; J. Berriat Saint Prix ; P. Boncenne ; G.J. Favard de Langlade; E.-N. Pigeau ; J. Curasson; C.-E. Delvincourt ; J. Grenier ; J.-B. Proudhon ; J.-J. Rolland de Villargues; C. Mollot; C. Renouard; A. Boucher D Argis ; G.-L. Carre ; F.-X. Garnier ; P. Bravard-Veyrieres ; A. Chauveau; D. et A. Dalloz; A. Duranton; J.-B. Duvergier; J.-G. Locre; G. Masse ; P.-A. Merlin de Douai ; J.M. Pardessus ; C. Toullier ; R.-T. Troplong. 
33. Ainsi, si l'on prend l'exemple de l'avocat Georges-Bonaventure Battur ${ }^{31}$, son ouvre est régulièrement citée dès la première période (I825-1835), puis connaît un important pic entre I836 et I858, où ses travaux sont référencés chaque année au recueil. De I859 à i88o, Battur reste encore fortement présent, même s'il l'est moins que sur les vingt années précédentes. Enfin, son référencement au recueil ne décroît véritablement qu'à partir de I88I, malgré un petit retour sur la période I89I-I902. Entre I903 et I9I4, son taux de référencement est au plus bas, même si l'auteur n'a pas encore totalement disparu des colonnes du Dalloz.

Schéma 2

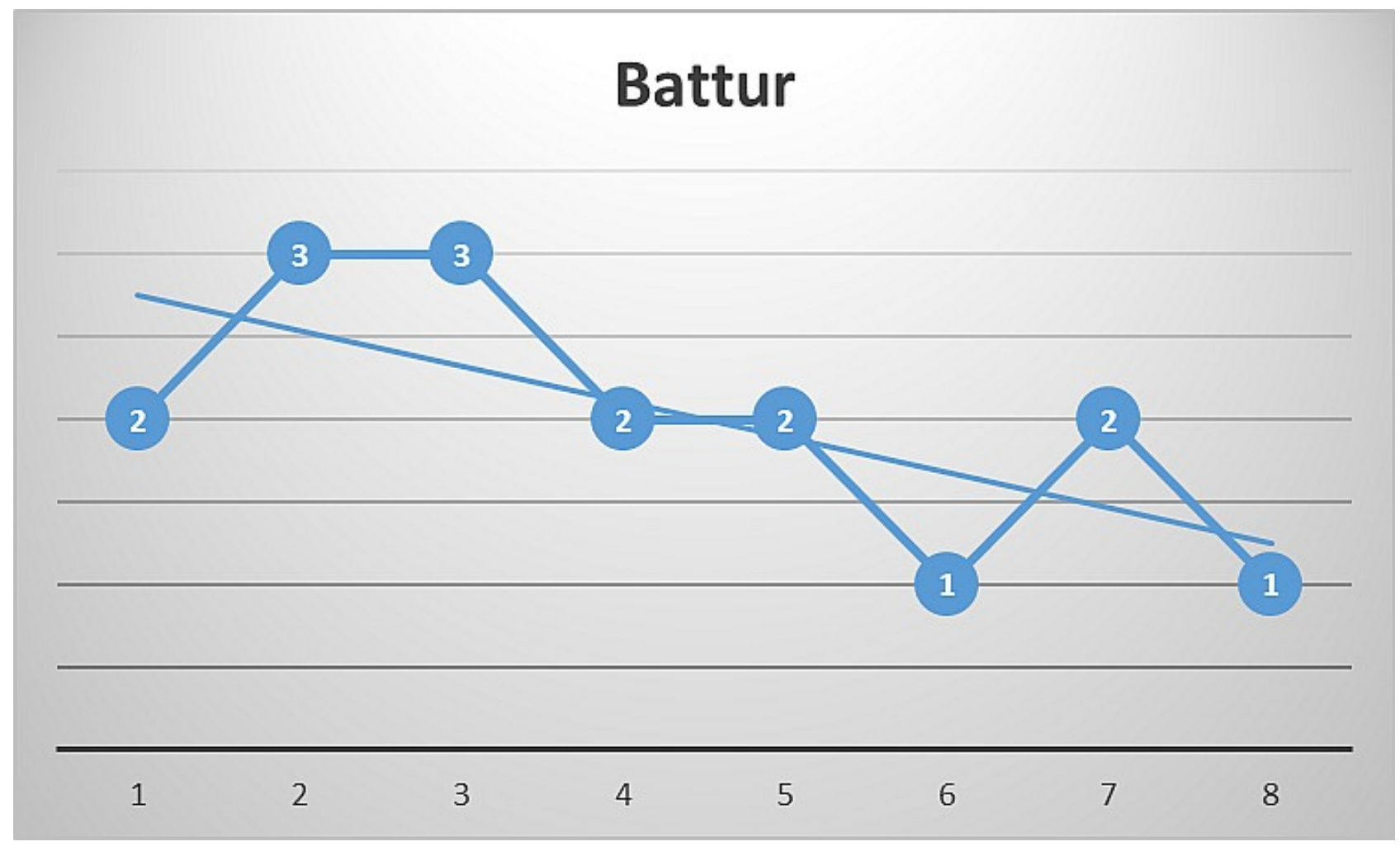

34. Sur l'ensemble de la période, la courbe de tendance bibliométrique de Battur au recueil Dalloz est donc décroissante (schéma 2); fortement cité jusqu'aux années I880, avec un pic au milieu du siècle, l'avocat ne semble plus faire autant autorité à partir de la Belle Époque, où ses apparitions seront de plus en plus rares. En cela, le cas de Battur est tout à fait représentatif des mutations de la pensée juridique au sein des recueils du XIX siècle.

35. Autrefois très nombreux et plébiscités, les travaux des praticiens se raréfient en effet progressivement à la Belle Époque, au profit des ouvres universitaires. Alors que les avocats, magistrats, notaires ou encore fonctionnaires de l'Enregistrement n'ont plus le temps ni les moyens de se consacrer à la rédaction ou à la mise à jour de leurs ouvrages ${ }^{32}$, les universitaires investissent au contraire pleinement le champ doctrinal, qui devient leur chasse-gardée. En produisant des travaux à un rythme et dans une proportion que les praticiens ne peuvent plus égaler, mais aussi en publiant dans tous les domaines du droit et de la littérature

3I Avocat à la Cour de Paris, Georges-Bonaventure Battur (I788-I850) a publié une vingtaine de travaux, dont plusieurs consultations, des essais sur la religion et la société, la politique et la diplomatie, ou encore d'intéressantes observations sur l'ordonnance royale du 24 mars i8I9 relative à la nouvelle organisation de l'École de droit de Paris. Le recueil Dalloz renvoie régulièrement à son Traité des privilèges et bypothéques (2 vol., Paris, Nève, I818) ainsi qu’à son Traité de la communauté de biens entre époux (2 vol., Paris, Barois et Duprat, 1830).

32 La professionnalisation des métiers du droit, l'instauration de la patente des avocats ou encore l'emballement et la complexification de la vie juridique et judiciaire expliquent en partie ce désengagement des praticiens de la « doctrine » à la fin du XIX ${ }^{\mathrm{e}}$ siècle. 
juridique - notamment dans les recueils d'arrêts autrefois détenus par les praticiens - les membres de l’École prennent alors le pas sur la pensée juridique. $\mathrm{La}$ « doctrine » entre en leur possession.

36. Cela ne signifie pas pour autant que les travaux universitaires se substituent immédiatement et intégralement à ceux des praticiens; si les références à des articles scientifiques (français comme étrangers), à des thèses ou encore à des «traités élémentaires » (généralement plus théoriques que pratiques) connaissent une croissance très sensible au recueil Dalloz à partir des années 1880 , le fond bibliométrique dominant demeure encore bien pourvu en ouvres de praticiens du droit, qu'elles soient anciennes ou nouvelles.

37. Ainsi, l'analyse bibliométrique permet de mesurer - et de pondérer - les changements qui se produisent dans la physionomie de la pensée juridique au XIXe siècle. Une lecture superficielle du recueil laissera au lecteur une nette (et exacte !) impression de «professoralisation » de la pensée juridique, qui débute aux alentours des années i88o, pour se consolider définitivement autour du fameux « Moment $1900{ }^{33}$; mais au-delà des apparences et des impressions, les données bibliométriques révèlent plus froidement que, parmi les I3I auteurs les plus fréquemment cités entre 1903 et I9I4 (indice 3), « seuls 》 6I d'entre eux sont des universitaires ${ }^{34}$, soit un taux d'environ $46,9 \%$. A titre de comparaison, sur la période la plus éloignée - I825-I835 -, ce même taux était certes plus faible, mais pas excessivement éloigné, atteignant les $38 \%$ ( 8 enseignants sur 2 I auteurs $)^{35}$.

1825-1835 (auteurs "Indice 3")

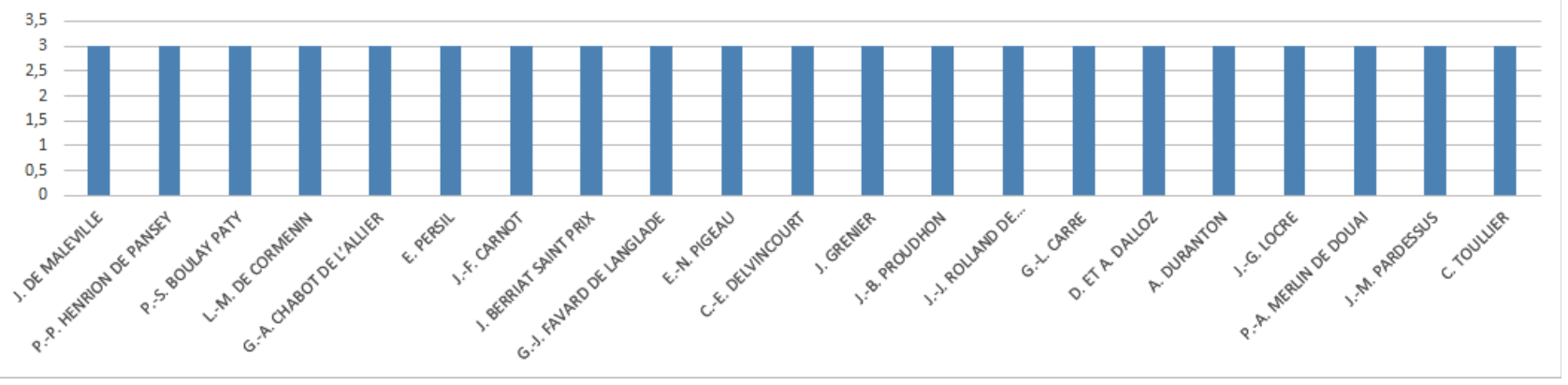

Nom incomplet sur le graphique ci-dessus : J.-J. Rolland de Villargues

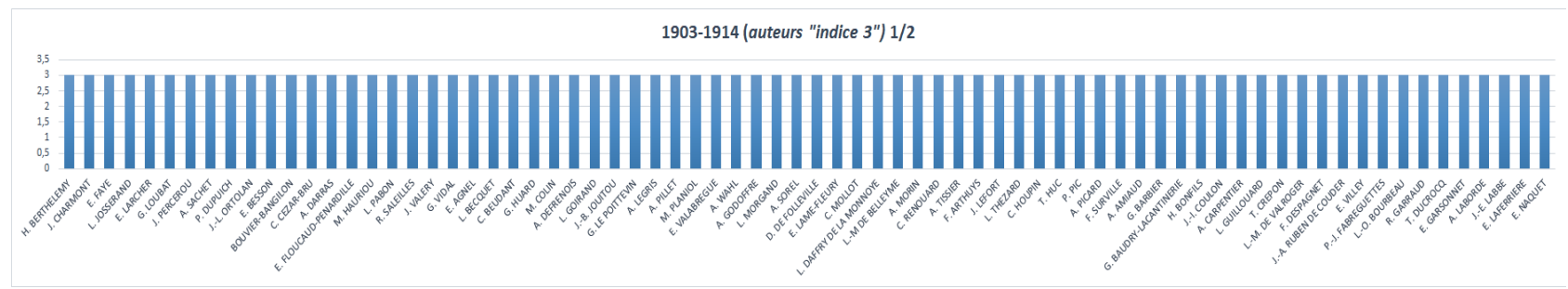

33 Voir O. Jouanjan et É. Zoller (dir.), Le «moment » 19oo. Critique sociale et critique sociologique du droit en Europe et aux États-Unis, Paris, Éditions Panthéon-Assas, 2015.

34 Il s'agit de: H. Berthelemy; J. Charmont ; L. Josserand ; J. Percerou ; J.-L. Ortolan ; C. Cezar-Bru ; M. Hauriou ; R. Saleilles ; J. Valery ; G. Vidal ; C. Beudant ; M. Colin ; G. Le Poittevin ; A. Pillet ; M. Planiol ; E. Valabregue ; A. Wahl ; D. de Folleville; A. Tissier; F. Arthuys; L. Thezard; P. Pic; F. Surville; G. Baudry-Lacantinerie; H. Bonfils; A. Carpentier ; L. Guillouard ; L.-M. de Valroger ; E. Villey ; L.-O. Bourbeau ; R. Garraud ; T. Ducrocq ; E. Garsonnet ; J.E. Labbe ; E. Laferriere ; E. Naquet ; G. Colmet Daage ; E. Thaller ; A. Weiss ; E.-D. Glasson ; A. Laurin ; C. Lyon-Caen ; L. Renault ; A. Boistel ; G.-L. Carre ; A. Desjardins ; F.-X. Garnier ; F. Laurent ; C. Aubry ; E.-L. Bonnier ; P. BravardVeyrieres ; A. Chauveau ; E. Colmet de Santerre ; C. Demangeat ; A.-M. et G. Demante ; C. Demolombe ; A. Duranton ; J.M. Pardessus ; A. Rodiere ; C. Toullier et A. Valette.

35 J. Berriat Saint Prix ; G.-L. Carre ; C.-E. Delvincourt ; A. Duranton ; J.-M. Pardessus ; E.-N. Pigeau ; J.-B. Proudhon et C. Toullier. 
1903-1914 (auteurs "indice 3") 2/2

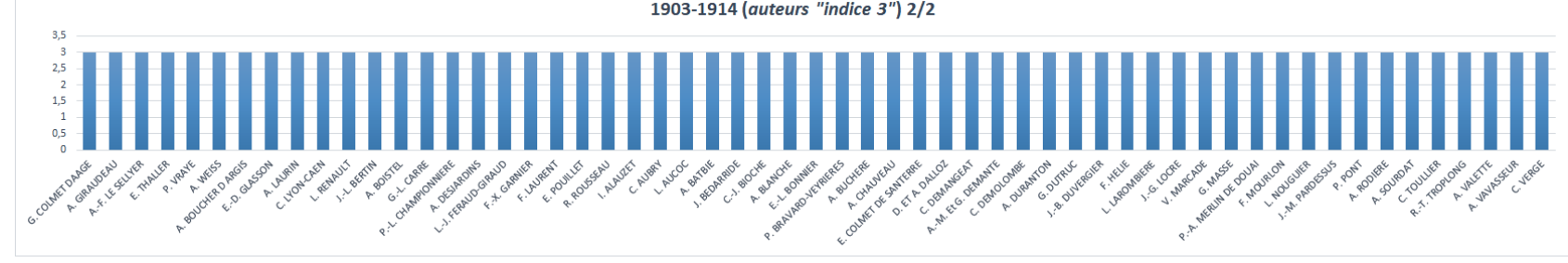

38. Le graphique regroupant les auteurs systématiquement référencés entre I825 et 1914 (schéma I) nous livre, pour l'essentiel, une cohorte de praticiens oubliés de l'historiographie de la pensée juridique : Battur, Bellot des Minières, Xavier Benoît, Bourguignon, Carnot, Chardon, Curasson, Daviel, Emerigon, Grün, Legraverend, Lepage, Magnin, Malapeyre et Jourdain, Mollot, Persil, Renouard, Tarbé, Tarrible, Tessier, Vazeille, Vincens, autant de noms et d'ouvres aujourd'hui effacés - ou presque -, de la mémoire juridique.

39. Ce tableau invite également à des études plus approfondies sur les travaux et sur l'impact d'auteurs mieux connus, tels que les praticiens Rolland de Villargues, de Cormenin, Grenier, Massé, Loiseau, ou les professeurs Bravard Veyrières, Malpel, Pigeau, Poncet et Thomine-Desmazures (père).

40. Il interroge enfin sur l'extraordinaire longévité d'un Berriat Saint-Prix, d'un Henrion de Pansey ou d'un Favard de Langlade, auteurs à la notoriété immense en leur temps, qu'il faudrait redécouvrir ; et que penser de la longévité d'un Delvincourt, dont l'œuvre, généralement reconnue comme médiocre, perdure pourtant jusqu’à la veille de la Grande Guerre?

4I. Parmi ses multiples intérêts, l'étude bibliométrique permet également de livrer des informations précieuses sur le genre d’ouvrages plébiscités par les rédacteurs - et indirectement, par les lecteurs, du recueil -.

42. Un rapide coup d'œil sur les 56r auteurs entrés dans la base de données donne déjà quelques pistes. Un grand nombre d'entre eux ont rédigé des traités « théoriques et pratiques » usuellement limités à une thématique ${ }^{36}$, ainsi que des études ou des commentaires de lois ou de réformes particulières ${ }^{37}$. Cette littérature « en patchwork », faite d'études affûtées sur des points de droit précis, semble caractéristique de la pensée juridique dominante au Dalloz sur l'essentiel du siècle ; une pensée juridique d'experts, moins soucieuse d'élaborations théoriques et de constructions intellectuelles générales, que d'analyses détaillées et de mises en pratiques du droit. En d'autres termes, cette pensée reflète davantage le droit tel qu'il était réellement praticable et mis en ouvre à l'époque, et apparaît ainsi plus « réaliste » qu’ « idéaliste ».

43. De nouveau, cela ne signifie nullement que la théorie soit négligée, comme le montre notamment - et pour ne citer qu'elle -, le succès de l'œuvre d'Aubry et Rau dans les colonnes du périodique. En outre, de nombreux commentateurs de lois ont une production littéraire qui dépasse la seule «technique juridique ». Pour reprendre l'exemple de Battur cité plus haut, l'avocat déploie ses conceptions politiques, économiques et sociales au sein de nombreux essais, publiés en marge de ses traités des Priviléges et de la Communautés $^{8}$. Catholique et légitimiste, la vision « sociétale » de lauteur doit naturellement être prise en compte dans l'analyse de son ouvre juridique, mais aussi dans le succès qu'elle a reçu. Ainsi, une pensée juridique technicienne ou experte ne signifie nullement une pensée dénuée de vision d'ensemble ; l'étude approfondie de ces auteurs, méconnus mais influents, permettrait donc de dessiner une image plus précise des juristes et de leur(s) culture(s) au XIX siècle.

36 Quelques exemples, parmi des centaines: J. Bédarride, Traité des faillites et banqueroutes; Traité des chemins de fer au point de vue du transport des voyageurs et des marchandises; O. Benech, Traité de l'emploi et du remploi de la dot; Daviel, Traité de la législation et de la pratique des cours d'eau.

37 Chez les mêmes auteurs: J. Bédarride, Commentaire de la loi du I7 juillet 1856; Commentaire des lois sur les brevets d'invention; O. Benech, Des tribunaux civils d'après la loi du II avril I838; A. Daviel, Commentaire de la loi du 29 avril 1845 .

38 Citons notamment son Traité de droit politique et de diplomatie (1824), De la religion catholique considérée comme une nécessité sociale (1826) et De l'Ordre et de la liberté (1829). 
44. À la fin du siècle, les grandes ouvres de l'École scientifique émergent évidemment au sein de la bibliométrie du recueil; néanmoins, les travaux d'expertise - encore bien souvent rédigés par des praticiens spécialisés dans un domaine ou un contentieux juridique -, demeurent toujours fortement référencés. Ainsi, un auteur aujourd'hui oublié comme le magistrat Adrien Sachet, qui publie en I899 la première édition de son imposant Traité théorique et pratique de la législation sur les accidents du travail, sera beaucoup plus souvent cité que les deux grands professeurs de législation industrielle, Georges Bry et Paul Pic, dont les traités sont aujourd'hui considérés comme des jalons de la pensée juridique travailliste ${ }^{39}$. Bien sûr, le niveau de référencement et la fréquence de citation d'une œuvre ne disent rien de sa véritable qualité ; toutefois, ils permettent de repérer de manière fiable son niveau de diffusion, sa popularité, en d’autres termes, ces indicateurs permettent de savoir si une œuvre était véritablement lue, connue, et sans doute pratiquée.

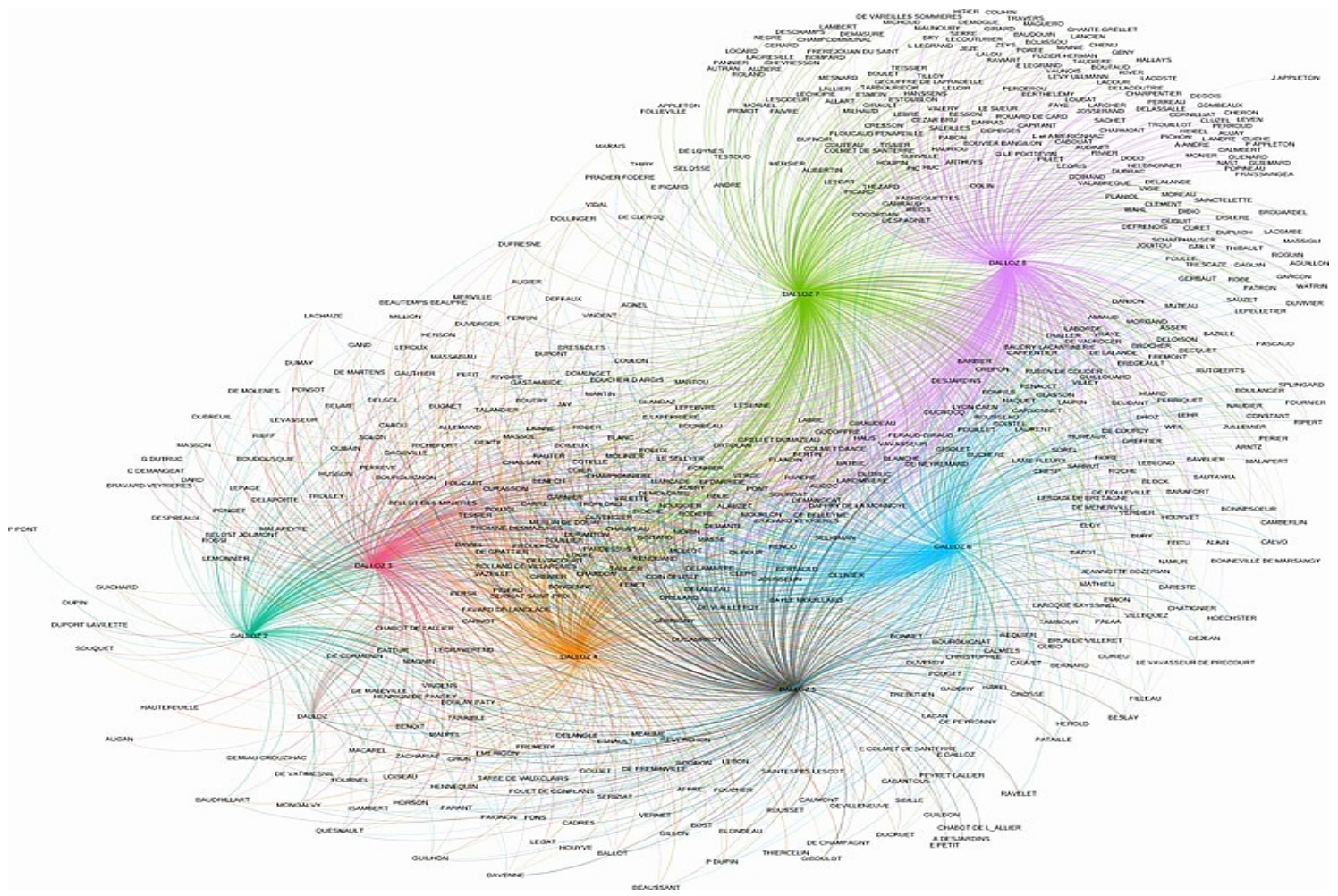

Graphique bibliométrique général du recueil Dalloz $(1825-1914)^{40}$

Légende: $:$ alloz = période 1825-1835; Dalloz2 = période 1836-1847; Dalloz 3 = période 1848-1858; Dalloz 4 = période 1859-1869; Dalloz $5=$ période 1870-1880; Dalloz 6 = période 1881-1891; Dalloz 7 = période 1892-1902 ; Dalloz 8 = période 1903-1914.

39 Adrien Sachet (1856-1826) est encore plus fortement référencé dans le recueil concurrent Sirey, où il publie trente notes d'arrêt sur les accidents du travail entre 1901 et 1925. Sur Georges Bry et sur Paul Pic, voir notamment G. Aubin et J. Bouveresse, Introduction historique au droit du travail, Paris, Presses universitaires de France, 1995; voir aussi N. Hakim, «Les premiers traités de législation industrielle et la jurisprudence : les ressources d'une influence doctrinale au Palais », dans Les acteurs de l'histoire du droit du travail, J.-P. Le Crom (dir.), Rennes, Presses universitaires de Rennes, 2004, p. 2939 ; Id., « La science de la question sociale de Paul Pic, ou les malheurs de l'hétérodoxie dans les facultés de droit », dans Le renouveau de la doctrine française - les grands auteurs de la pensée juridique au tournant du XX siècle, N. Hakim et F. Melleray (dir.), Paris, Dalloz, spécialement p. I23-I24.

40 Ce graphique a été généré par le logiciel Gephi. Il contient l'ensemble des 56I auteurs, pondérés par leur niveau de référencement, et reliés aux différentes périodes sur lesquelles ils sont cités. Plus les auteurs sont référencés sur une longue période, plus ils se rapprochent du centre du graphique; à l'inverse, les auteurs excentrés sont les auteurs les moins référencés, ou référencés seulement sur une courte période. Le « fond bibliométrique » du recueil est donc composé des auteurs regroupés en « $\mathrm{U} \gg$ au centre du graphe. 
45. Confirmant les observations statistiques ci-dessus analysées, ce dernier graphique a l'avantage d'offrir une représentation globale et instantanée de la bibliométrie du recueil, qu'un tableau de données ou même qu'un ensemble de courbes ne sauraient rendre de manière aussi immédiate et explicite.

46. Une évidence, qui n'en semble pourtant pas une lorsque l'on se penche sur les études historiographiques: les grands auteurs de l' «École scientifique » n'apparaissent qu'en minorité et en marge, dans le coin haut-droit du graphique (Dalloz 8 et 7$)^{41}$, au milieu d'une nuée d'autres contemporains oubliés. Ainsi, Bufnoir Saleilles, Esmein, Planiol, Josserand - pour ne citer qu'eux -, côtoient des Lallier, Rouard de Card, Tilloy, Boulet, Trescaze, Couteau, là-encore quelques noms pris au hasard, mais tout aussi bien référencés - sinon mieux -, que les grands professeurs.

47. Autre élément intéressant, le cour du graphique, celui qui regroupe les auteurs les plus cités sur le siècle, forme une constellation de noms célèbres, moins célèbres ou inconnus, et illustre parfaitement la diversité de la prose juridique plébiscitée par les rédacteurs (et indirectement, par les lecteurs) du recueil Dalloz. Aux côtés des ouvrages théoriques et civilistes de Toullier, Duranton, Troplong ou Demolombe, figurent une multitude d'études plus ciblées, comme celles de Faustin Hélie, de Grenier, de Carré, de Cotelle, de Sourdat, de Gastambide, de Richefort... Un peu plus en marge encore, mais référencées sur plusieurs périodes, les cuvres spécialisées et parfois pionnières de Jeannotte-Bozérian sur la bourse et les agents de change, celles de Louis Sarrut sur les chemins de fer ou encore celles d'Alphonse Grün sur l'assurance-vie ${ }^{42}$. Ce coeur bibliométrique montre ainsi qu'aucun sujet, de la question des sépultures (De Vuillefroy) à celle des assurances maritimes (Émerigon, Boulay-Paty), en passant par le droit notarial (Rolland de Villargues), ne pouvait échapper au contentieux, à la réflexion et à la plume des praticiens. Nous sommes alors bien loin de la « monomanie » du Code civil, abusivement dénoncée chez les auteurs du XIX siècle.

48. Précisons, enfin et à nouveau, que ces 56I auteurs ne sont que la « face immergée de l'iceberg », rescapés d'un premier filtre bibliométrique qui pourrait - devrait - encore être affiné, et qui dévoilerait ainsi de nouveaux noms et de nouvelles ouvres.

\section{Conclusion(s)}

49. Bien que sommaire, cette analyse bibliométrique démontre qu'une grande part des auteurs véritablement lus et plébiscités au XIX siècle sont tombés dans les limbes d'une histoire de la pensée juridique, essentiellement centrée - pour ne pas dire « autocentrée »-, sur la doctrine, sur l' «École ». Il est peut-être important, aujourd'hui, de s'interroger sur la nature de la pensée juridique dont nous faisons l'histoire, et sur les méthodes que nous mettons en place pour la passer au tamis.

50. Cherchons-nous à connaître le véritable impact que les auteurs et leurs ouvres ont pu laisser dans la pensée et (surtout ?) dans la pratique de leur siècle ? Cherchons-nous à savoir comment les juristes se documentaient pour assurer leurs offices, vers quels ouvrages ils se tournaient, quels auteurs avaient leurs faveurs, et pour quelles raisons?

5I. Si les belles « cathédrales » doctrinales sont les plus séduisantes pour l'intellect, étaient-elles forcément les plus représentatives de la pensée juridique quotidienne, étaient-elles celles des hommes du métier, celles

4I Il s'agit bien entendu des deux dernières périodes de l'étude, qui s'étendent de I892 à I9I4.

42 J.-F. Jeannotte-Bozerian, La bourse, ses opérateurs et ses opérations, appréciés au point de vue de la loi, de la jurisprudence et de l'économie politique et sociale, suivi d'une indication des réformes les plus urgentes, 2 vol., Paris, É. Dentu et A. Marescq, I859; L. Sarrut, Législation et jurisprudence sur le transport des marchandises par chemins de fer, Traité théorique et pratique, Préface par Ch. Lyon-Caen, Paris, A. Chaix, I874; A. Grün (et Joliat), Traité des assurances terrestres et de l'assurance sur la vie des hommes, Paris, Chez les auteurs, 1828 . 
qu'ils connaissaient, achetaient, prenaient le temps de lire, d'annoter, et conservaient sur les étagères de leurs bibliothèques?

52. Les colonnes du recueil Dalloz ne sont pas le réceptacle fidèle et unique de la pensée juridique du $\mathrm{XIX}^{\mathrm{e}}$ siècle; elles n'en sont qu'une page, dont l'analyse statistique ramène des berges de l'oubli ces « petites mains du droit » à la notoriété et à la longévité qui surprennent aujourd'hui. L'expérience bibliométrique effleurée ici mériterait, nous le croyons, d'être approfondie et étendue à dautres revues, d'autres médias juridiques. Avant d'aborder la «pensée juridique » en tant que telle, n'est-il pas naturel d'en baliser les terrains, d'en identifier les acteurs les plus significatifs, les plus lus et, possiblement, les plus influents de leur temps?

53. La bibliométrie n'est pas une fin en soi, mais un préliminaire; elle s'inscrit dans une méthodologie d'identification et de classification préalable des auteurs et des œuvres. Ce n'est qu'une fois les référencements et les statistiques effectués, le lectorat cible identifié, les médias et réseaux de diffusion mis à jour, que l'analyse intellectuelle des ouvrages pourra véritablement révéler la - ou probablement les pensées juridiques dominantes d'une époque, au sein de classes de juristes mieux identifiés.

54. Si ce travail préalable est fastidieux, et s'il nécessite d'importantes prospections dans des domaines a priori éloignés de l’analyse de la pensée - notamment, la maîtrise des outils et des méthodes bibliométriques, ou encore l'histoire de l'édition et des réseaux de diffusion du livre et de la presse juridiques-, il nous semble néanmoins indispensable pour cartographier la pensée juridique contemporaine, pour mieux en saisir les acteurs et les développements. Ainsi, nous serons davantage en mesure de savoir de quelle pensée juridique nous faisons vraiment l'histoire.

\section{Pierre-Nicolas BARENOT}

